Check for updates

Cite this: New J. Chem., 2020, 44, 15300

DOI: 10.1039/d0nj90102d

rsc.li/njc

\section{Correction: Solid-phase extraction followed by deep eutectic solvent based dispersive liquid- liquid microextraction and GC-MS detection of the estrogenic compounds in wastewater samples}

\author{
Reza Davoodi, ${ }^{a}$ Ramin Nabizadeh Nodehi, ${ }^{\text {ab }}$ Noushin Rastkari, ${ }^{\text {b }}$ \\ Ali Akbar Zinatizadeh, ${ }^{\complement}$ Amir Hossein Mahvi*ade and Nazir Fattahi ${ }^{\dagger}$
}

Correction for 'Solid-phase extraction followed by deep eutectic solvent based dispersive liquid-liquid microextraction and GC-MS detection of the estrogenic compounds in wastewater samples' by Reza Davoodi et al., New J. Chem., 2020, 44, 9844-9851, DOI: 10.1039/D0NJ00911C.

The authors regret that an acknowledgement was omitted from the original article. The authors would like to thank the Center for Water Quality Research, Institute for Environmental Research, Tehran University of Medical Sciences for financial support of this research study as Grant number 48964. The authors also regret that an affiliation was missing from the original manuscript, which should have been listed as affiliation $d$ : The author Amir Hossein Mahvi is also affiliated to the Center for Water Quality Research (CWQR), Institute for Environmental Research (IER), Tehran University of Medical Sciences, Tehran, Iran. The original affiliation $d$, the Center for Solid Waste Research, should be listed as affiliation $e$. The corrected list of affiliations is shown above.

The Royal Society of Chemistry apologises for these errors and any consequent inconvenience to authors and readers.

\footnotetext{
${ }^{a}$ Department of Environmental Health Engineering, School of Public Health, Tehran University of Medical Sciences, Tehran, Iran. E-mail: ahmahvi@yahoo.com

${ }^{b}$ Center for Air Pollution Research (CAPR), Institute for Environmental Research (IER), Tehran University of Medical Sciences, Tehran, Iran

${ }^{c}$ Department of Applied Chemistry, Faculty of Chemistry, Razi University, Kermanshah, Iran

${ }^{d}$ Center for Water Quality Research (CWQR), Institute for Environmental Research (IER), Tehran University of Medical Sciences, Tehran, Iran

${ }^{e}$ Center for Solid Waste Research (CSWR), Institute for Environmental Research (IER), Tehran University of Medical Sciences, Tehran, Iran

${ }^{f}$ Research Center for Environmental Determinants of Health (RCEDH), Kermanshah University of Medical Sciences, Kermanshah, Iran. E-mail: n.fattahi@kums.ac.ir; Fax: +98 8338263048; Tel: +989183364311
} 